\title{
INSIDE THE USCAP JOURNALS
}

doi:10.1038/labinvest.2016.67

\section{MODERN PATHOLOGY}

\section{The NEPTUNE scoring system}

See page 671

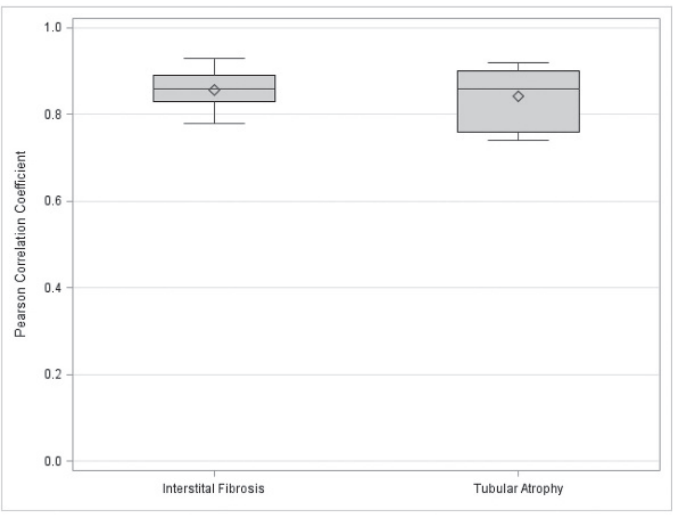

Barisoni et al assessed the efficacy of NEPTUNE (the multicenter Nephrotic Syndrome Study Network) by taking 12 pathologists ( 8 from NEPTUNE and 4 non-NEPTUNE) with varying experience and analyzing their concordance in assessment of glomerular descriptors. The NEPTUNE pathologists generally showed higher concordance than the non-NEPTUNE pathologists did, although this was not the case across every variable. Excellent concordance was seen for both interstitial fibrosis and tubular atrophy, independent of the number of years of observer experience. Digital technology, including software for annotation of glomeruli, offers the opportunity to systematically eliminate glomerular selection bias and increase interobserver concordance. The authors' goal was to provide a protocol with which success could be measured against the contribution to outcome and therapeutic guidance. This clinical foundation would thus provide the rationale for using this novel protocol in the classification of renal structures across multiple international consortia.

\section{Morphological staging with genotyping for lung cancer \\ See page 735}

Schneider et al tackled the complex distinction between primary cancers and intrapulmonary metastases in patients with multifocal lung cancer (a feature recently shown to comprise up to $20 \%$ of cases) by determining the role of morphology and genotype in staging surgically resected multifocal non-small cell lung cancer. With the results of existing methods being interpreted freely by pathologists in each center, and without defined features to assess or a standard procedure for incorporating molecular studies into

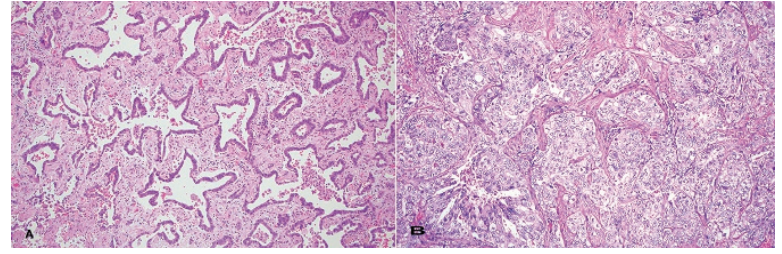

staging, there was room for a more standardized method. The authors illustrate the difficulty by showing two nodules of lung adenocarcinoma with different morphological features, thought to represent two separate, independent primary tumors, but genotyping showed that they had the same KRAS mutation, favoring the analysis that they represent intrapulmonary metastases. While uncertain that different mutations definitively identify separate primary cancers, the authors support the use of genotyping in combination with morphological data in order to improve clinical staging.

\section{LABORATORY INVESTIGATION}

\section{Wound contraction by fibroblast migration}

See page 741
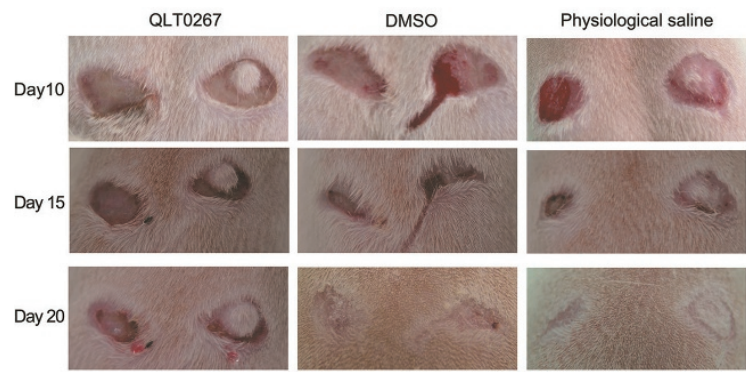

Li et al demonstrated that integrin-linked kinase (ILK) could play a role in cutaneous wound contraction, with ILK and PI3K/AKT inhibitors inhibiting wound contraction and re-epithelialization and delaying wound healing in vivo. They injected an ILK inhibitor around wounds, calculated the percentage of wound closure, and conducted scratch wound-healing and transwell assays. They used these assays to investigate the role of human skin fibroblast migration in wound healing and the effects of the ILKPI3K/AKT pathway in this process. Treatment with $5 \mu \mathrm{M} I \mathrm{LK}$ inhibitor and $50 \mu \mathrm{M}$ PI3K/AKT inhibitor attenuated activity in human skin fibroblasts. The authors concluded that the ILK/AKT pathway probably mediates transforming growth 
factor- $\beta 1$-induced expression of $\alpha$-smooth muscle actin as well as myofibroblast differentiation in dermal fibroblasts, through the influence of fibroblast migration and fibroblast-to-myofibroblast differentiation. Future research will establish the potential clinical impact of these inhibitors.

\section{Sarcoma explant tissue culture \\ See page 752}

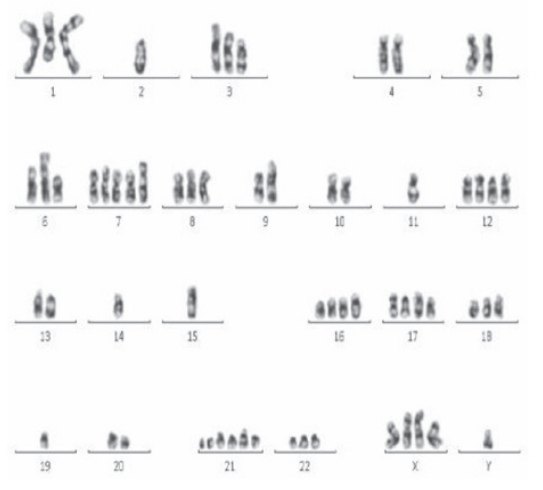

As a tool for preclinical research into various cancers, cell culture is a crucial method in achieving a better understanding of disease as well as in the development of new therapeutics. Given the heterogeneity and rarity of sarcomas, there is a scarcity of patient-derived cell lines. Over nearly a decade, Muff et al collected 734 tissue samples from which cells were successfully isolated and classified as 'growing.'The group established the relationship between malignancy and time to cell culture growth, with cells from bone sarcomas having significantly shorter times to reach sufficient growth for freeze storage compared with soft-tissue sarcoma. The authors' hypothesis regarding the low success rate for culturing liposarcoma is that the high fat content of the cells in suspension prevents plate adherence. The possibility of freezing tissue prior to culture allows the sharing of rare patient material and the expansion of a library of sarcoma cell types in culture for use in preclinical studies and personalized medicine.

\section{nature.com/pathology}

\section{Genomic landscape of liver cancer}

Fujimoto et al analyzed 300 liver cancers from Japanese individuals to catalog somatic alterations across the genome, identifying point mutations, structural variations, and virus integrations in coding and noncoding regions. Their analyses added new signatures to those reported in a previous French study, overlapping when it came to smoking and alcohol consumption. They were also able to identify new driver genes for liver cancer; chromatin regulators (ASH1L), nuclear receptor signaling genes (MACROD2), and liver metabolism genes (HNF4A), while confirming known driver genes (TERT, RPS6KA3, RB1, ARID1A, PTEN). Several lincRNAs are

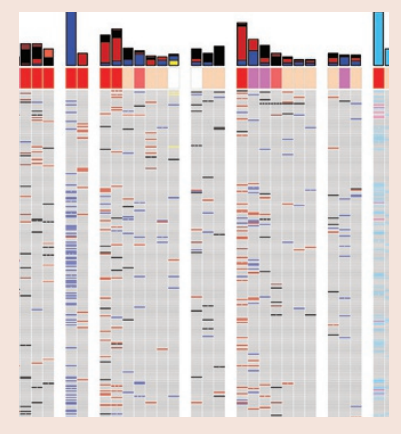
induced by cellular stress such as hypoxia, and cell proliferation or invasion is promoted by the upregulation of genes such as MALAT1. The identification of structural variations that correlate in position with oncogenes with elevated expression and also with suppressed expression levels of tumor-suppressor genes suggests that these structural variations can promote liver carcinogenesis by epigenetic means.

Nature Genetics 2016:48:500-509; doi:10.1038/ng.3547

\section{New mutation in uveal melanoma}

Uveal melanomas are the most common tumor of the eye. Moore et al analyzed genomic data from 136 uveal melanoma samples and found rare, but recurrent, mutations in CYSLTR that were mutually exclusive from the common GNAQ and GNA11 mutations seen in these tumors. Since uveal melanomas do not exhibit BRAF, NRAS, KIT or NF1 mutations as cutaneous melanomas do, a new signature needed to be identified. The authors' data suggest that CYSLTR2 is a uveal

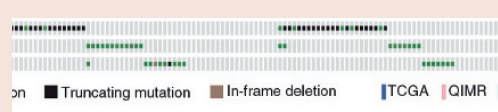
melanoma oncogene and could play a critical role in $\mathrm{G}_{\mathrm{aq}}$ signaling. It is involved in leukotriene-mediated signaling, in both inflammation and fibrosis. Knockdown of CYSLTR2 in cells expressing Leu129GIn exhibited decreased expression of protein as well as of melanocyte-lineage genes. Given the highly attractive nature of G-protein-coupled receptors as drug targets because of their location on plasma membranes and their availability for high-throughput cell-based screens for drug discovery, the authors propose the development of high-affinity'inverse agonists'.

Nature Genetics, published online 18 April 2016; doi:10.1038/ng.3549

\section{Unique genomics of ERCC2 mutated urothelial tumors}

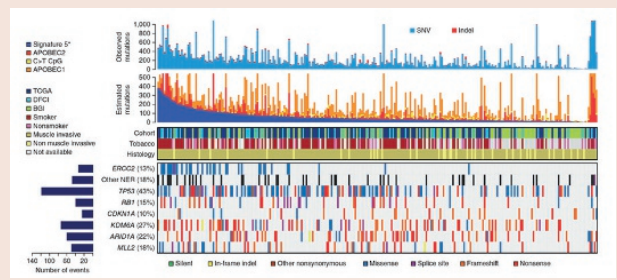

Kim et al examined the mutational processes in urothelial cancer to assess a specific mutational signature associated with somatic alterations in the nucleotide-excision repair (NER) pathway, specifically in the ERCC2 gene. A series of genetic signatures were identified, one of which showed correlation with a previously characterized COSMIC signature 5 . In these tumors, ERCC2 mutations appear to be the most common genetic event driving the increase in lowerfidelity repair. The ERCC2 mutations occur early in tumorigenesis, indicating the need for further investigation into the role this gene plays in bladder tumor biology. A seemingly modest correlation between this new pattern (termed signature $5^{*}$ ) and smoking was also identified, possibly reflecting lower-fidelity repair of tobacco-mediated DNA damage in these patients. The authors' plan is to further investigate signature $5^{*}$ activity in tumors without an ERCC2 mutation in order to better understand how this signature occurs. Nature Genetics, published online 25 April 2016; doi:10.1038/ng.3557

Emma Judson contributed to these reviews. 